\title{
Analysis of Buck-Boost Converter Inductor Loss Using a Simple Online B-H Curve Tracer
}

\author{
H.Y. Chung, F.N.K. Poon, C.P. Liu and M.H. Pong \\ Power Electronics Laboratory \\ Department of Electrical and Electronic Engineering \\ The University of Hong Kong \\ PokFulam Road, Hong Kong \\ Email: hychung1@eee.hku.hk
}

\begin{abstract}
A simple method to plot online B-H curve and calculate core loss of the inductor in a conventional buck-boost converter is developed. In order to obtain a reliable loss data, measurement error and phase shift error are analyzed and then quantified. A new method to minimize measurement error is given. A new core loss model in terms of switching duty cycle is presented. This model predicts variation of core loss of an inductor operating in various conditions in a switching converter. This inductor model can be generalized to inductors in other switching converter topologies.
\end{abstract}

\section{INTRODUCTION}

Miniaturization of power converters requires minimization of components on the printed circuit board. Magnetic components consume a lot of space. Pushing switching frequency higher enables a design engineer to choose a smaller size magnetic core in his design so that minimization of magnetic components can be achieved. However, pushing frequency higher and higher results in increasing losses of magnetic components. The losses are core loss and copper loss. In this paper the main concern is to investigate core loss of an inductor in a switching power converter.

Core loss comprises hysteresis loss, eddy current loss and residual loss. Hysteresis loss is directly proportional to switching frequency and square of magnetic flux density. Eddy current loss in a magnetic core is similar to eddy current loss inside a copper conductor so it is directly proportional to both square of switching frequency and square of magnetic flux density. Residual loss is directly proportional to both frequency and magnetic flux density [1].

Core loss can be read from the loss graph on a magnetic data sheet. However, information provided by the manufacturer is often incomplete. The designers sometimes cannot find the core loss under the switching frequency and flux level they desire. Another method to obtain core loss is by referring to loss formula given in the form of $C_{1} \times B^{C_{2}} \times f^{C_{3}}$. The precision of $\mathrm{C}_{1}, \mathrm{C}_{2}$ and $\mathrm{C}_{3}$ is not high enough and error in calculation will be high. Another point is that loss formula or loss graph presented on data sheet is obtained when the core is under sinusoidal voltage excitation. In a power electronics circuit, usually voltage across an inductor is a square wave but not a sinusoidal one. Thirdly, the parameters of a core sample vary from sample to sample due to manufacturing tolerance. For the three reasons given above, loss data acquired from the information given on a data sheet may not lead to an accurate core loss. Measurement techniques for magnetic cores have been reported in the literatures [1] [2] [3] [4] and [5]. Reference [1] suggests a method which can avoid the notorious phase shift error introduced in the electrical measurement. In reference [2], potential sources of measurement errors are discussed along with circuits suitable for high-frequency sine-wave or square-wave excitation. In reference [3] an experimental method to measure losses in magnetic components is presented. Reference [4] develops an automatic method to derive loss formula directly by measuring a set of loss data using electrical method. Reference [5] describes the development and verification of measurement techniques for the design of high-frequency SMPS transformers. All of them perform the core loss measurement in a standalone way, i.e. when the magnetic core is not put into a real operating circuit. When magnetic cores are put onto a real operating circuit, their core losses may be different from the result obtained from standalone test.

In this paper, a simple online B-H curve tracer is developed. It is capable to plot B-H curve while the inductor is in circuit and simultaneously calculate core loss. This setup provides information on whether the inductor is well designed or not. Saturation of core is also noticeable by referring to the B-H curve plotted. Core loss during saturation is more than that when the core is operating at linear region so we should always avoid operating the core in saturation region. When an inductor is operated in the saturation region, core temperature will increase. The increase in temperature decreases maximum flux density flowing without saturating the core [6]. As a result the core becomes more saturated. This positive loop continues until the power converter fails to work.

Electrical measurement has the benefit of easy and repeatable measurement but it is less accurate than calorimetric method. Reference [7] points out that electrical measurement can lead to large error. Core loss of a DC to DC power converter with output power of several tens of Watts is 
usually less than $1 \mathrm{~W}$. It is therefore worth investigating the sources of errors and then finds way to minimize them.

Core loss predicted by formula $C_{1} \times B^{C_{2}} \times f^{C_{3}}$ has been used extensively for its clearance and simplicity. However, $C_{1}, C_{2}$ and $C_{3}$ for each core are found when the core is under sinusoidal excitation. In a switching power converter, voltage across an inductor is usually square in shape. Reference [8] finds out that core loss can increase a lot for large difference between the on and off time. In the present work we propose that core loss can be represented by the formula $C_{1} \times B^{C_{2}} \times f^{C_{3}} \times D^{C_{4}} \times(1-D)^{C_{3}}$, which includes the influence of duty cycle $D$.

\section{ANALYSIS OF MEASUREMENT AND PHASE SHIFT ERRORS}

Core loss of ferrite core used in high frequency power converter is usually small, often in the order of one watt. However power level of a converter is of several tens of watts. In an electrical measurement, core loss is found as the difference between input power to inductor and output power from it. The input power and output power are large compared with core loss. As a result the error can be large and a detailed analysis of measurement and phase shift error is essential.

Any inductors working in continuous mode are excited by a bipolar square voltage source, with the voltage and current waveforms shown in Fig. 1. If there is no loss, integral of voltage current product across one period equals to zero (1). In a lossy inductor, the result of the integral will be equal to its core loss.

$$
\int_{0}^{D T} V_{1}(t) i_{1}(t) d t+\int_{D T}^{T} V_{2}(t) i_{2}(t) d t=0
$$

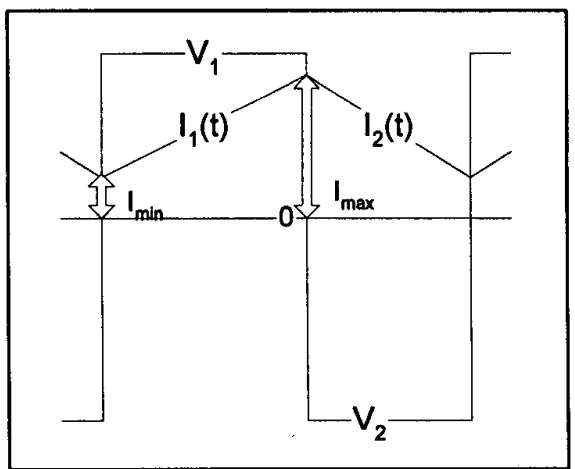

Fig. 1.Voltage and current of an inductor of a power converter working in continuous mode

Fig. 2 show that the measurement system contains several source of errors. There are measurement errors and phase shift errors. Measurement errors include errors from voltage measurement and current measurement. These measuring errors depend on which measuring apparatus are used. Phase shift error is due to signal time difference between the voltage measurement system and the current measurement system. This error is notorious and has been pointed out in papers [1] and [2].

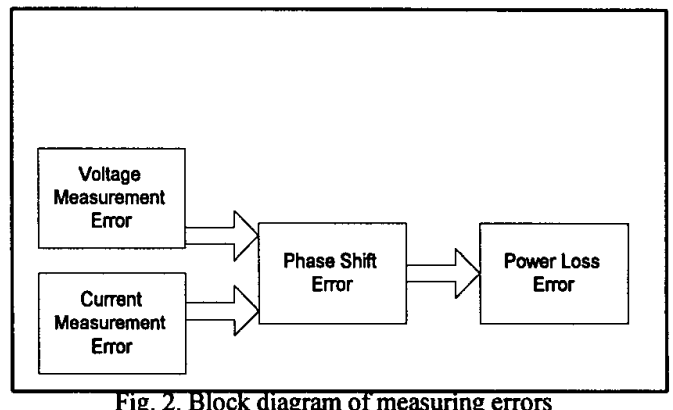

There are several assumptions made in the investigation. In the analysis of measuring errors, it is assumed there is no distortion of voltage and current waveform and only DC offset errors are present. It is also assumed the average DC errors of $V_{1}, V_{2}, I_{1}$ and $I_{2}$ to be $\Delta V_{1}, \Delta V_{2}, \Delta I_{1}$ and $\Delta I_{2}$ respectively. Fig. 3 shows the measurement errors. The measurement error is given by,

$$
\begin{aligned}
& \text { Measurement Error }=\frac{\Delta V_{1}}{T} \int_{0}^{D T} i_{1}(t) d t+\frac{\Delta V_{2}}{T} \int_{D T}^{T} i_{2}(t) d t \\
& +\Delta I_{1} D\left(V_{1}+\Delta V_{1}\right)+\Delta I_{2}(1-D)\left(V_{2}+\Delta V_{2}\right)
\end{aligned}
$$

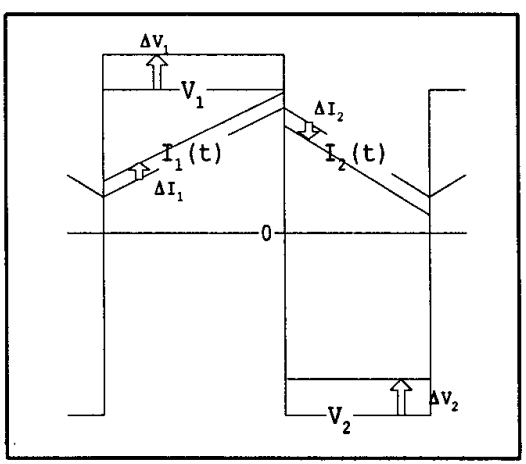

Fig. 3. Measurement error

The sign of each quantity should be noted. Fig. 3 shows that $V_{1}$ is positive while $V_{2}$ is negative. $\Delta I_{1}$ is positive while $\Delta \mathrm{I}_{2}$ is negative. Assume the errors are $2 \%$ of its measured value and let $V_{1}, V_{2}$, average $I_{1}$ and average $I_{2}$ over one period be $30 \mathrm{~V},-30 \mathrm{~V}, 1 \mathrm{~A}$ and $1 \mathrm{~A}$ respectively and $\mathrm{D}=0.5$. $I_{\max }=1.5 \mathrm{~A}$ and $I_{\min }=0.5 \mathrm{~A}$. In the worse case shown in Fig. $3, \Delta \mathrm{V}_{1}$ and $\Delta \mathrm{V}_{2}=0.6 \mathrm{~V}$ and $\Delta \mathrm{I}_{1}=0.03 \mathrm{~A}$ and $\Delta \mathrm{I}_{2}=-0.03 \mathrm{~A}$. The maximum absolute error becomes $1.5 \mathrm{~W}$. At the other extreme, $\Delta \mathrm{V}_{1}, \Delta \mathrm{I}_{1}$ and $\Delta \mathrm{V}_{2}$ are negative and $\Delta \mathrm{I}_{2}$ are positive, absolute error is $-1.5 \mathrm{~W}$. Core loss is often smaller than $1 \mathrm{~W}$ 
under excitation voltage of $30 \mathrm{~V}$ and switching frequency of several hundred $\mathrm{kHz}$. In order to obtain a reasonable accuracy, the measuring system has to be able to give errors less than $0.2 \%$ of the measured value.

Another source of error is phase shift error. It is caused by signal time delay between the current measuring system and the voltage measuring system. Fig. 4 shows the phase shift between current and voltage of an inductor of a power converter. Here the current waveform is modelled as straight line, then we have phase shift error given by,

$$
\text { Phase Shift Error }=\frac{V_{1}-V_{2}}{T} \int_{D T}^{D T+\Delta t} i_{2}(t) d t-\frac{V_{1}-V_{2}}{T} \int_{0}^{\Delta t} i_{1}(t) d t
$$

whencurrent leads voltage by $\Delta t$

$$
\text { Phase Shift Error }=\frac{V_{1}-V_{2}}{T} \int_{T-\Delta t}^{T} i_{2}(t) d t-\frac{V_{1}-V_{2}}{T} \int_{D T-\Delta t}^{D T} i_{1}(t) d t
$$$$
\text { when current lags behind voltage by } \Delta t
$$

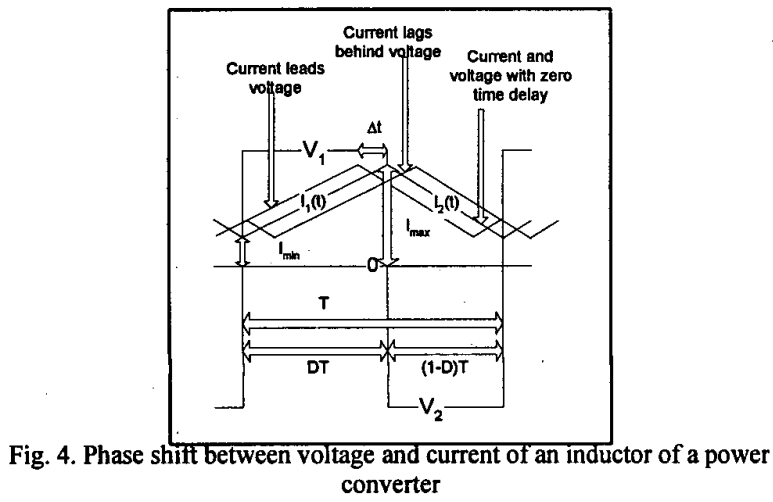

where $\Delta t$ is the time shift between voltage and current. The error will be greater than zero when current leads voltage and become negative when current lags behind voltage. If $D=$ $0.5, V_{1}=30 \mathrm{~V}, \mathrm{~V}_{2}=-30 \mathrm{~V}$, average $\mathrm{I}_{1}=$ average $\mathrm{I}_{2}=1 \mathrm{~A}, \Delta \mathrm{t}=10 \mathrm{~ns}, \mathrm{~T}=5 \mu \mathrm{s}, \mathrm{I}_{\min }=0.5 \mathrm{~A}$ and $\mathrm{I}_{\max }=1.5 \mathrm{~A} . \Delta \mathrm{t}$ is only $1 / 500$ of period $T$. The error is $0.1198 \mathrm{~W}$ if current leads voltage. When current lags behind voltage, phase shift error becomes $-0.1198 \mathrm{~W}$. When $\Delta t=20 \mathrm{~ns}$ or $1 / 250$ of period $\mathrm{T}$, phase shift error becomes $\pm 0.2395 \mathrm{~W}$. Again this cannot be neglected since core loss is often less than $1 \mathrm{~W}$.

\section{MEASUREMENT SETUP}

Our target magnetic component is the inductor of a conventional buck-boost converter. It is shown in Fig. 5. Fig. 6 shows the measurement setup. The input voltage is $30 \mathrm{~V}$. The inductor core under test is an EFD20, 3F3 core, airgap of $0.05 \mathrm{~mm}$ exists along all limbs of the core, inductance of $60 \mu \mathrm{H}$ with effective length of $47 \mathrm{~mm}$, effective area of $31 \mathrm{~mm}^{2}$ and $N_{s}=N_{p}=20$. The equivalent resistance of the current sensing resistors is $0.516 \Omega$. The small current sensing resistance causes little disturbance to the testing circuit. The oscilloscope used is Tektronix TDS320. The temperature variation of the inductor under investigation is small, from $30^{\circ} \mathrm{C}$ to $40^{\circ} \mathrm{C}$. As a result the temperature effect on core loss is ignored in the measurement.

Values of Magnetic flux density $B$ and magnetic field intensity $\mathrm{H}$ are required to plot the online B-H curve. Secondary winding with the same number of turns as primary is wound on the inductor. Secondary voltage is sensed.

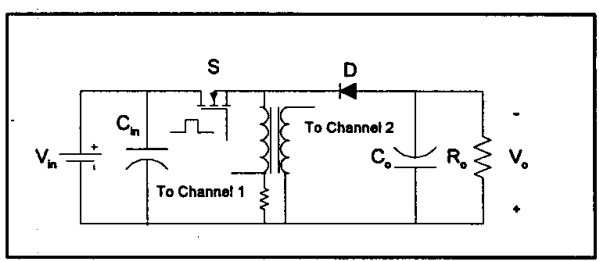

Fig. 5. A Conventional buck-boost converter

The alternating magnetic flux density inside inductor core is governed by Faraday's law,

$$
B(t)=\frac{1}{N A e} \int v_{s}(t) d t
$$

where $\mathrm{N}$ is the number of inductor turns and $\mathrm{A}_{\mathrm{c}}$ is the effective area of the magnetic core.

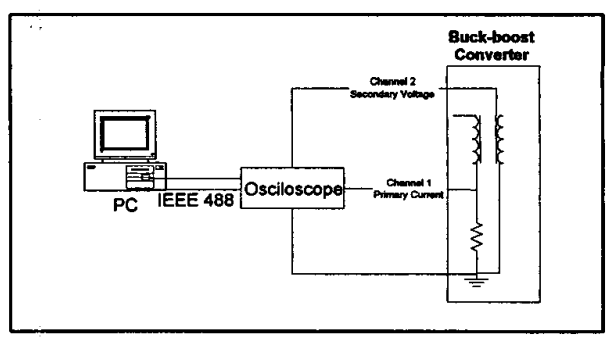

Fig. 6. Core loss measurement setup

Measuring secondary voltage bears two advantages. First of all, electrical isolation between power circuit and voltage measuring system, typically an oscilloscope, is achieved. In case where both two terminals of inductor being measured are not grounded, electrical isolation is essential. Secondly, measuring secondary voltage eliminates the effect of resistance of primary winding acting upon measured loss.

Primary current is sensed with the aid of current sensing resistors. From the principle of Ampere's Law we have,

$$
H(t)=\frac{N i(t)}{l e}
$$


where $\mathrm{N}$ is the number of turns and $\mathrm{l}_{\mathrm{e}}$ the effective length of the magnetic core. B-H curve can be plotted on the computer screen. The next step is to calculate core loss. Two electrical methods can be used to measure core loss of the inductor. The first method is by multiplying primary current and secondary voltage of the inductor under measurement. That gives instantaneous power. The average of the instantaneous power over one switching period gives core loss,

core loss $=\frac{V_{1}}{T} \int_{0}^{D T} i_{1}(t) d t+\frac{V_{2}}{T} \int_{D T}^{T} i_{2}(t) d t$

where $\mathrm{D}$ is the duty cycle and $\mathrm{T}$ is the switching period. The second method requires computation of the enclosed area of the $\mathrm{B}-\mathrm{H}$ curve. Core loss is obtained by multiplying that area by switching frequency and effective volume of the inductor core. Finding area involves integration which means more tedious calculation work has to be performed. Also in this case error cannot be analysed easily. Due to these two reasons the first method is adopted to calculate core loss. Loss found from these two methods are equivalent and is verified in appendix.

To facilitate fast data capturing, B-H curve plotting and calculation of core loss, a Pentium personal computer with program written in LabVIEW is developed during research. The program has several functions and is shown in table 1.

TABLE I

FUNCTIONS OF LabVIEW PROGRAM Functions of LabVTEW Program

\begin{tabular}{|ll|}
\hline Input & Functions of LabVIEW Program \\
& $\begin{array}{l}\text { Core effective area } \\
\text { Core effective length } \\
\text { Number of secondary inductor turns } \\
\text { Value of current sensing resistance }\end{array}$ \\
\hline Data processing & $\begin{array}{l}\text { Calculate B and H using (5) and (6) } \\
\text { Calculate core loss using (7) }\end{array}$ \\
\hline Output & $\begin{array}{l}\text { Display online B-H curve } \\
\text { Show core loss }\end{array}$ \\
\hline
\end{tabular}

In the measurement setup, voltage measurement system is formed by a voltage probe connected to an oscilloscope. There are many combinations of equipment that can function as a current measurement system. The first is a current sensing resistor, a voltage probe and an oscilloscope. The second is an AC current probe connected to a digital oscilloscope and the third setup is a DC current probe, connected to an amplifier circuit and then joined to a digital oscilloscope. Using current probe has the advantages of better electrical isolation, better noise immunity and minimum disturbance to the testing circuit. However they have poor frequency response, which means there is distortion in the measured current waveform. Secondly the introduction of current probe causes time delay between voltage measurement system and current measuring system and results in notorious phase shift error. As a result it is not recommended to use a current probe to get current waveform. A voltage probe connected to an oscilloscope and the current sensing resistors are used as a current measuring system.

\section{MINIMIZATION OF ERRORS IN MEASURING SETUP}

Measurement errors have been analyzed in section II. It is found from (2) and (3) that considerable error can be incurred in electrical measurement. Therefore it is a must to find ways to reduce errors in measurement.

The first method used to minimize the measurement error is voltage error offset technique. The voltage is taken from secondary winding of the inductor which is free from the effect of primary winding resistance. Volt-time integral of secondary voltage across one period is equal to zero. Refer to Fig. 1, numerically,

$\frac{1}{T} \int_{0}^{D T} V_{1}(t) d t+\frac{1}{T} \int_{D T}^{T} V_{2}(t) d t=D V_{1}+(1-D) V_{2}=0(8)$

Because there exists errors $\Delta V_{1}$ and $\Delta V_{2}$ in voltage measurement,

$D\left(V_{1}+\Delta V_{1}\right)+(1-D)\left(V_{2}+\Delta V_{2}\right)=C$

where $\mathrm{C}$ is any real number. After rearrangement, we have,

$D\left(V_{1}+\Delta V_{1}-C\right)+(1-D)\left(V_{2}+\Delta V_{2}-C\right)=0$

then,

$D\left(\Delta V_{1}-C\right)+(1-D)\left(\Delta V_{2}-C\right)=0$

A program written in LabVIEW can calculate the value $C$ and subtract it from the voltage measured. The first two terms of (2) becomes,

$\frac{\Delta V_{1}-C}{T} \int_{0}^{D T} i_{1}(t) d t+\frac{\Delta V_{2}-C}{T} \int_{D T}^{T} i_{2}(t) d t$

where $i_{1}(t)$ and $i_{2}(t)$ are rising and falling current waveform without errors respectively. Then it is obvious that (12) equals to zero automatically.

Error introduced by the voltage measurement system (the first two terms in (2)) vanishes. The remaining error is the error caused by the current measuring system. In our system it is a voltage probe connected to an oscilloscope and current sensing resistors. 
Measurement Error

$=\Delta I_{1} D\left(V_{1}+\Delta V_{1}-C\right)+\Delta I_{2}(1-D)\left(V_{2}+\Delta V_{2}-C\right)$

$=\left(\Delta I_{1}-\Delta I_{2}\right) D\left(V_{1}+\Delta V_{1}-C\right)$ by $(10)$

If the current measurement channel on the oscilloscope is set to $0.1 \mathrm{~V} / \mathrm{Div}$. Coupling method is selected as AC. As a result there is no need to adjust the vertical scale when DC current of the inductor changes. Assume the inductor maximum peak to peak current is $1.2 \mathrm{~A}$, or $0.612 \mathrm{~V}$ measured from the voltage probe since the value of the current sensing resistors is $0.516 \Omega$. Under these conditions, $D C$ accuracy found from the instruction manual of Tektronix TDS320 is $\pm 0.0224 \mathrm{~V}$ under measuring condition [9]. That means about 0.2 divisions. 0.2 divisions are recognizable. The actual error of either $I_{1}$ or $I_{2}$ in the measurement can be $\pm 0.0224 \mathrm{~V}$ but their difference should be less than 0.2 divisions. Assume $\Delta \mathrm{I}_{1}$ $-\Delta \mathrm{I}_{2}=|0.01|$ which is 0.1 divisions in the oscilloscope screen. $\mathrm{V}_{1}=30 \mathrm{~V}$. Then according to (13) and assume $\Delta \mathrm{V}_{1}-\mathrm{C}$ be very small, maximum measurement error when $\mathrm{D}=0.3,0.4,0.5$, 0.6 and 0.7 are $0.09,0.12,0.15,0.18$ and $0.21 \mathrm{~W}$ respectively. It can be seen that the larger is the duty cycle, the larger will be its maximum error.

Phase error is minimized, since current sensing resistors and a voltage probe are used as the current measurement system. A $200 \mathrm{kHz}$ square wave generated by a HP8116A signal generator is applied to two voltage probes of channel 1 and channel 2 . The delay is found to be very close to zero when time base is set to $5 \mathrm{~ns} / \mathrm{Div}$. As a result phase shift error is set to be zero.

The above analysis shows that the maximum error of the measuring system is $0.09,0.12,0.15,0.18,0.21 \mathrm{~W}$ when $\mathrm{D}=$ $0.3,0.4,0.5,0.6$ and 0.7 respectively under the measuring conditions, which is acceptable in the case concerned.

\section{RESULTS}

Core loss is related to frequency $f$ and magnetic flux density B. It is commonly used in industry to represent core loss by Steinmetz law,

$$
C_{1} \times B^{C_{2}} \times f^{C_{3}}
$$

where $C_{1}, C_{2}$ and $C_{3}$ are constant. The core under test is excited by sinusoidal voltage. In a switched mode power supply, the excitation waveform is usually bipolar squarewave voltage. One of the parameters used to characterize bipolar square-wave voltage is the duty cycle. According to [8], the less symmetrical is the magnetizing current, the more will be the core loss. It is therefore proposed that core loss can be represented by a new model in terms of duty cycle,

$$
C_{1} \times B^{C_{2}} \times f^{C_{3}} \times D^{C_{4}} \times(1-D)^{C_{3}}
$$

where $C_{1}, C_{2}, C_{3}, C_{4}$ and $C_{5}$ are constants. After collecting a number of core loss data, multiple linear regression technique [10] is used to perform the curve fitting. When DC current flowing through the inductor is $1 \mathrm{~A}$, and frequency ranges from $150 \mathrm{kHz}$ to $300 \mathrm{kHz}$, magnetic flux density $B$ is up to $70 \mathrm{mT}$ and $\mathrm{D}$ is from 0.3 to 0.7 . Core loss in the inductor under investigation (EFD20, 3F3) before saturation is given by,

$$
8.289 \times 10^{-7} \times B^{1.923} \times f^{1.503} \times D^{-0.512}(1-D)^{-0.585}
$$

where the error is estimated in section four.

Refer to (16), we can see core loss becomes minimum when duty cycle is about 0.5 . In other words the less symmetrical is the magnetizing current, the larger will be the core loss with $\mathrm{B}$ and $\mathrm{f}$ kept constant. Then $C_{1} \times B^{C_{2}} \times f^{C_{3}}$ is constant and $D^{-0.512} \times(1-D)^{-0.585}$ as a function of $\mathrm{D}$ is plotted in Fig. 7. $D^{-0.512} \times(1-D)^{-0.585}$ can reflect the relative magnitude of core loss incurred. The result agrees with that found in [8].

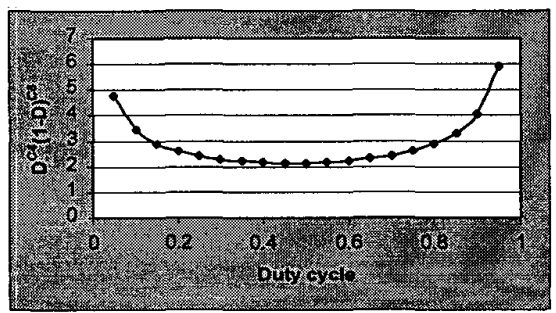

Fig. $7 D^{-0.512} \times(1-D)^{-0.585}$ as a function of duty cycle and flux is constant

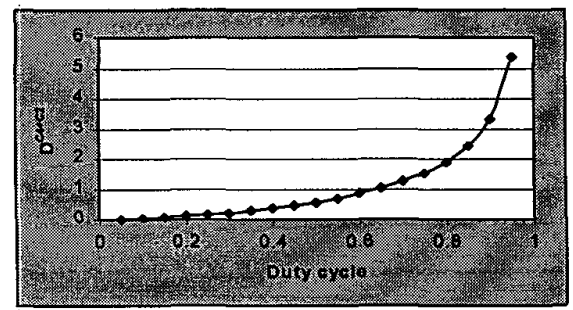

Fig. 8. $D^{C_{1}+C_{2}} \times(1-D)^{C_{3}}$ as a function of duty cycle and input voltage is constant

If only duty cycle is changed but input voltage remains constant, magnetic flux density will vary. By (20) and (21), magnetic flux density is proportional to time or its duty cycle. Doubling duty cycle will double magnetic flux density. $C_{1} \times f^{C_{3}}$ is constant in this case. $B^{C_{2}} \times D^{C_{4}} \times(1-D)^{C_{3}}$ is equal to $C_{6} \times D^{C_{4}+C_{2}} \times(1-D)^{C_{3}}$ so 
that $D^{C_{4}+C_{2}} \times(1-D)^{C_{s}}$ can reflect the relative magnitude of core loss incurred. In Fig. 8, $D^{C_{4} C_{2}} \times(1-D)^{C_{3}}$ is plotted against duty cycle. We can see that increasing duty cycle alone will increase core loss and vice versa.

At the first glance of (16), it can be seen that the higher the switching frequency is, the higher will be the core loss. However, the magnetic flux density B of an inductor in a switching power converter is inversely proportional to frequency $f$. Doubling the frequency has the effect of halving magnetic flux density. The exponent of magnetic flux density is larger than that of frequency. The net effect is that increasing switching frequency alone will decrease core loss instead of increasing it while reducing switching frequency alone will increase core loss. In this case, $C_{1} \times D^{C_{4}} \times(1-D)^{C_{3}} \quad$ is a constant. Then $B^{C_{2}} \times f^{C_{3}}=C_{7} \times f^{C_{3}-C_{2}}$ since $\mathrm{B}$ is inversely proportional to $f$. As a result we can represent relative magnitude of core loss by the factor $f^{C_{3}-C_{2}} \cdot f^{C_{3}-C_{2}}$ or $f^{-0.42}$ as a function of normalized frequency is plotted in Fig. 9.

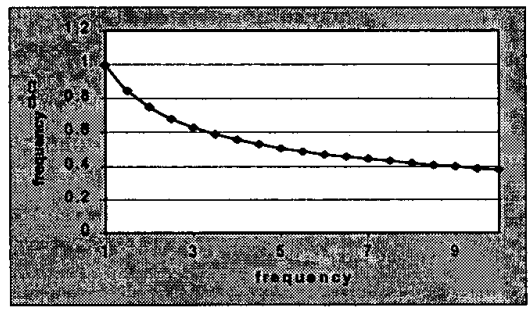

Fig. 9. $f^{C_{3}-C_{2}}$ as a function of normalized frequency

Fig. 10 and 11 represent graphically core loss vs magnetic flux density $B$ when $D=0.5, f=200 \mathrm{kHz}$ and $\mathrm{D}=0.4$ and $\mathrm{f}=$ $240 \mathrm{kHz}$ respectively. The calculated core loss (solid line) and measured core loss (diamond) agree with each other well.

Since the role of inductors in other switching converter topologies, such as buck, boost and forward converters are similar. The inductor model given by (15) can be applied.

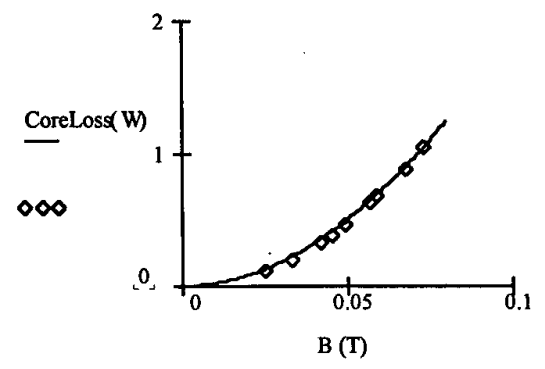

Fig.10. Core loss vs flux density when $D=0.5$ and $f=200 \mathrm{kHz}$

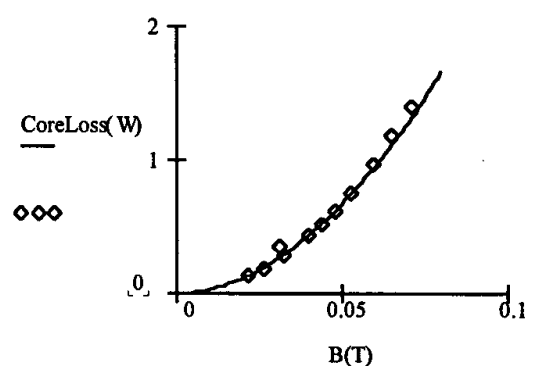

Fig. 11. Core loss vs flux density when $D=0.4$ and $f=240 \mathrm{kHz}$

The B-H curve tracer so developed can plot the B-H curve online. Therefore it is easy for a designer to notice whether the core is saturated or not. Two figures shown below are the B-H curve being plotted when the core is working in linear region and in saturation region.

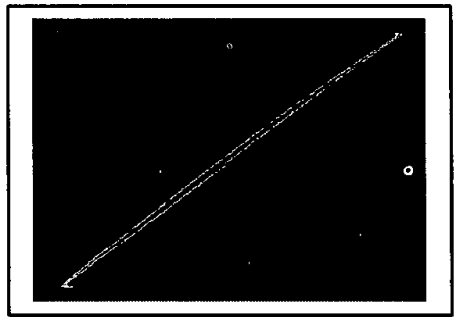

Fig. 12. B-H curve of the inductor of a buck-boost converter working in linear region

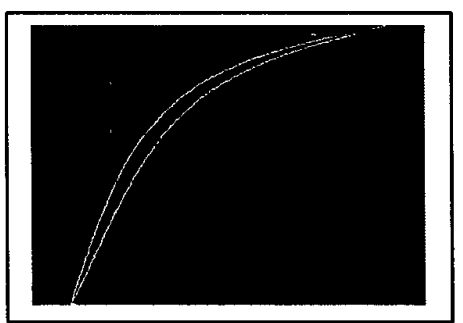

Fig. 13. B-H curve of the inductor of a buck-boost converter working in saturation region

\section{CONCLUSION}

A measuring system to plot $\mathrm{B}-\mathrm{H}$ curve and measure online core loss of an inductor in a conventional buck-boost converter is developed. The system can give instantaneous core loss data when the core is operating. Various measurement errors are present in the system. They are analysed and is found to be large if the data are not handled properly. A new method is proposed to minimize the error and finally a reasonable error is acquired. Using the B-H curve tracer, core loss of an inductor (EFD20, 3F3) core is obtained. It is proposed that core loss of an inductor can be 
represented by a new model in terms of duty cycle. The core losses predicted by the model agree with measured core losses well. The new core loss model can be extended to inductors in other switching converter topologies.

\section{APPENDIX}

The energy density stored in the magnetic field plus the energy density stored in the material is given by: [11]

$$
E=\int_{0}^{B} H d B
$$

$H$, the magnetic field intensity is a function of $B$, the magnetic flux density. $B$ is a function of time $t$. Using expression (1), the energy density supplied to a magnetic core to increase its magnetic flux density from zero to value $B_{1}$ is given by:

$$
E=\int_{0}^{B_{1}} H(B) d B
$$

By Faraday's law, the voltage induced in a conductor is proportional to the change in the magnetic flux passing through the surface enclosed by that conductor.

For the inductor in a buck-boost converter, when the switch is ON, we have:

$$
V_{1}=N A_{e} \frac{\Delta B}{\Delta t}
$$

where $V_{1}$ the input voltage to the inductor, $N$ the number of inductor turns and $\mathrm{A}_{\mathrm{e}}$ the effective area of the magnetic core.

$$
B_{1}=B_{1}-0=\Delta B=k t
$$

where $k=\frac{V_{1}}{N A_{e}}$

substitute (20) into (18) and using power $P=\frac{d E}{d t}$, we have Instantaneous power density when the switch is $\mathrm{ON}$

$$
\begin{aligned}
& =P(t) \\
& =\frac{d E}{d t} \\
& =\frac{d}{d t} \int_{0}^{B_{1}} H(B) d B \\
& =k H(B) \\
& =\frac{V_{1}}{N A_{e}} \frac{N i(t)}{l_{e}} \\
& =\frac{V_{1 i}(t)}{A_{e} l_{e}}
\end{aligned}
$$

Average power density supplied to an inductor when the switch is ON

$$
=\frac{V_{1}}{A e l_{e}}\left(i_{m \mathrm{i}}\right)
$$

where $i_{m 1}$ is the average primary current when the switch is ON.

Similarly average power density returned from an inductor when the switch is OFF

$=-\frac{V_{2}}{\text { Ale }_{e}}\left(i_{\mathrm{m} 2}\right)$

where $i_{m 2}$ is the average primary current when the switch is OFF.

Average power loss density of the inductor

$=\frac{V_{1 i m 1}+V_{2 i m 2}}{\text { Aele }}$

Average power loss of the inductor

$=V_{1 i m_{1}}+V_{2} i_{m 2}$

$=\frac{V_{1}}{T} \int_{0}^{D T} i_{1}(t) d t+\frac{V_{2}}{T} \int_{D T}^{T} i_{2}(t) d t$

\section{REFERENCES}

[1] F. Dong Tan, Jeff L. Vollin and Slobodan M. Cuk, "A Practical Approach for Magnetic Core-Loss Characterization," IEEE Transactions on Power Electronics, Vol. 10, No. 2, March 1995

[2] V. Joseph Thottuvelil, Thomas G. Wilson and Harry A. Owen, JR, "High Frequency Measurement Techniques for Magnetic Cores", IEEE Transactions on Power Electronics, vol. 5, no. 1, January 1990.

[3] Jan Abraham Ferreira and Jaccobus D. van Wyk, "Experimental Evaluation of Losses in Magnetic Components for Power Converters," IEEE Transaction on Industry Applications, Vol. 27, No. 2, 1991

[4] Adalberto J. Batista, Joao Carlos S. Fagundes and Philippe Viarouge, "Automated System for Core Loss Measurement and Characterization: A Useful Tool for High Frequency Magnetic Components Design," Proceedings. ISIE 1998, pages 540 to 545 .

[5] David K. Conroy and Geoge F. Pierce, "Measurement Techniques For the Design of High-Frequency SMPS Transformers," APEC 1988 Conference Proceedings, pages 341 to 351

[6] J.J Rousseau, L. Zegadi and P. Tenant, "A Complete Hysteresis Model for Soft Ferrites Taking Into Account the Temperature," PESC 1996, Volume 1, pages 322 to 326 .

[7] T.G. Imre, W.A. Cronje, J.D. van Wyk and J.A. Ferreira, "Experimental Validation of Loss Calculations for a Planar Inductor," PESC 1999, volumel, pages 586 to 591

[8] M. Albach, Th. Durbaum and A. Brockmeyer, "Calculating Core Losses in Transformers for Arbitrary Magnetizing Currents A Comparison of Different Approaches," PESC 1996, Volume 2, pages $1463-1468$

[9] Tektronix TDS310, TDS320 and TDS350 Two Channel Oscilloscopes, Instruction Manual, pages 4-3 to 4-4.

[10] Ronald E. Walpole and Raymond H. Myers, Probability and Statistics for Engineers and Scientists Fifth Edition, Macmillan 1993, Chapter 12.

[11] Robert M. Fano, Lan Jen Chu and Richard B. Adler, "Electromagnetic Fields, Energy, and Forces," John Wiley and Sons, Inc. 1960, pages 285 to 289 